\title{
Benign Ovarian Cysts with Raised CA-125 Levels: Do We Need to Evaluate the Fallopian Tubes?
}

\author{
Devesh Sharma ${ }^{1}$ Anjali Vinocha ${ }^{1}$ \\ ${ }^{1}$ Department of Biochemistry, Laboratory Medicine, Delhi State \\ Cancer Institute, Delhi, India
}

J Lab Physicians:2020;12:276-280

\begin{abstract}
Address for correspondence Dr. Anjali Vinocha, MD, Department of Lab Medicine, Delhi State Cancer Institute, Dilshad Garden, Delhi 110095, India (e-mail: wadhawananjali2412@gmail.com).
\end{abstract}

\begin{abstract}
Keywords

- ovarian cyst

- CA 125 antigen

- ovarian neoplasm

Objectives It is not clearly known whether some benign (simple) ovarian cysts can convert into cancerous cysts. Size of cyst and wall abnormalities do predict the potentiality of malignancy. Not many studies have been done to explore the malignant potential of large-sized $(>5 \mathrm{~cm}$ ) unilocular ovarian cysts without wall abnormalities. This study evaluated the correlation between ultrasonographic size of benign ovarian cysts and carbohydrate antigen 125 (CA-125) levels.

Methodology Sixty (60) premenopausal women were recruited for the study preoperatively, based on transvaginal ultrasound (TVUS) findings present in the case record sheet received along with the CA-125 sample in the biochemistry laboratories. Those cases with elevated CA-125 levels were selected, where patients had unilocular ovarian cysts without wall abnormalities. CA-125 was done using ECLIA methodology (Cobas e411, Germany). Statistical correlation was calculated between the ovarian cyst size and CA-125 levels using Spearman's Rho coefficient.

Results Mean age group of subjects were $29.7 \pm 7.3$ years and mean value of CA-125 (normal < $35 \mathrm{IU} / \mathrm{mL}$ ) was found to be increased: $118.0 \pm 147.1 \mathrm{IU} / \mathrm{mL}$ so was the mean diameter of cysts (cut off $\leq 5 \mathrm{~cm}$ ): $48.6 \pm 59.8 \mathrm{~cm}$. No correlation was found between CA-125 levels and volume of ovarian cyst $(r=0.005, p=0.680)$ for all subjects.

Conclusions The lack of correlation between size of ovarian cysts and CA- 125 levels provides a hint that the ovarian cyst epithelium does not directly express CA-125 and it may come from sites like the fallopian tube. Thus, raised level of CA-125 in benign ovarian cyst should be followed-up more closely, demanding assessment of fallopian tubes for early diagnosis of ovarian cancer. Also, algorithms can be explored to include size of ovarian cyst and CA 125 levels to predict ovarian cancer.
\end{abstract}

\section{Introduction}

Ovarian cyst is a common condition. Globally, around 7\% of women are diagnosed with ovarian cysts at some point in their life. ${ }^{1}$ Ovarian cysts can be of two types: functional ovarian cysts (follicular cysts and corpus luteum cyst) which are small in size $(<3 \mathrm{~mm})$ and develop as part of the normal menstrual cycle and keep appearing and disappearing. The other type of ovarian cyst is nonfunctional and persistent. These are pathological ovarian cysts which include polycystic ovarian syndrome, endometriotic cyst, mucinous cystadenoma, serous cystadenoma, dermoid cyst, and paraovarian cysts. $^{2}$
DOI https://doi.org/ $10.1055 / \mathrm{s}-0040-1722547$ ISSN 0974-2727.
(C)2020. The Indian Association of Laboratory Physicians.

This is an open access article published by Thieme under the terms of the Creative Commons Attribution-NonDerivative-NonCommercial-License, permitting copying and reproduction so long as the original work is given appropriate credit. Contents may not be used for commercial purposes, or adapted, remixed, transformed or built upon. (https://creativecommons.org/licenses/by-nc-nd/4.0/).

Thieme Medical and Scientific Publishers Pvt. Ltd. A-12, 2nd Floor, Sector 2, Noida-201301 UP, India 
Mostly, ovarian cysts are asymptomatic and incidentally found during ultrasound. But large persistent cysts that that occur in approximately $8 \%$ of premenopausal women are often problematic and present with symptoms such as dull aching abdominal pain, abnormal uterine bleeding, fullness bloating in abdomen, difficulty in urination and, in severe cases, there may occur twisting or rupture of ovarian cyst, which may lead to an episode of fainting. ${ }^{2}$ Ovarian cysts are present in around $13 \%$ of postmenopausal women, and it requires careful workup and follow-up, as they may be precursor for ovarian carcinomas, which are usually diagnosed very late due to them being asymptomatic in the early stage. ${ }^{3}$

Carbohydrate antigen 125 (CA-125) is a member of mucin 16 family transmembrane glycoproteins which are found on ocular surface, respiratory tract ${ }^{4}$ and sections of female genital tract of Mullerian duct origin (uterus, fallopian tube, upper part of vagina). ${ }^{5}$ Its upper limit in healthy individuals is $35 \mathrm{U} / \mathrm{mL}$. Primary FDA-approved use of CA-125 involves monitoring of epithelial ovarian tumor. Apart from ovarian cancer, it is elevated in carcinomas of endometrium, lung, pancreas, breast, and colorectal. Benign conditions where it is raised include early pregnancy, hepatitis, cirrhosis, endometriosis, and pericarditis. CA-125 is therefore used to differentiate benign versus malignant pelvic mass such as adnexal masses and ovarian cysts. ${ }^{4}$

Invasive workup like biopsy is avoided in ovarian cysts to prevent spillage of tumor cells in case of malignancy. ${ }^{6}$ Therefore, noninvasive investigations like ultrasonography (USG) and CA-125 levels are mostly used in conjunction for this purpose. USG features of the cyst, which increase suspicion of malignancy, include multilocular cysts, solid areas, bilateral lesions, ascites and intra-abdominal masses. These USG features along with CA-125 levels and menopausal status are also included in the RMI algorithm (risk of malignancy index) for risk identification for ovarian cancer. ${ }^{7}$

Many strategies have been explored to improve the clinical usefulness of CA-125 for screening or early detection of ovarian cancer by improving its sensitivity and specificity. These include combining this investigation with transvaginal sonography, serial monitoring of CA-125 levels, and using multimarker panels like CEA and CA 19.9. ${ }^{6}$ But to the best of our knowledge, ovarian cyst size has not been evaluated till now for this matter. CA-125 alone is raised in only 20 percent of ovarian cancers; therefore, this study was planned to explore the possibility of including ovarian cyst size in existing diagnostic algorithms (including CA-125) like RMI, in order to increase their positive predictive probability.

\section{Methods}

\section{Study Design and Subjects}

Sixtypremenopausal patients were recruited. The women were considered premenopausal if they had regular mensuration going on. These patients were among those whose sample was collected for measuring CA-125 levels to evaluate ovarian cyst. As per the protocol of the laboratory, the patients case record sheets accompanied the test requisition form. Using the data from the case record sheet, only those patients were included in the study whose detailed recent ultrasound report (less than 1 week old) was available and there was finding of abnormal sized unilocular ovarian cyst $(>5 \mathrm{~cm})$ without wall abnormality. Both bilateral and unilateral cysts were included. The protocol of the study conformed to the ethical guidelines of the 1975 Declaration of Helsinki and it was approved by Institutional Ethical Committee for Human Research. An informed consent was obtained from all participants. Interviews were conducted and patients' medical files were studied to collect baseline demographic information, medical history and menstrual history. To exclude patients with raised CA-125 due to other conditions, our exclusion criteria included nonovarian neoplasms, including endometrial, pancreatic, lung, breast, and colorectal or other gastrointestinal tumor, women in the follicular phase of the menstrual cycle, and benign conditions such as cirrhosis, hepatitis, endometriosis, pericarditis and early pregnancy. ${ }^{5}$

\section{Power of Study}

To the best of our knowledge, no study has been conducted to evaluate correlation between the size of benign ovarian cyst and CA-125 levels, so for this sample size, we could not assess the power of study.

\section{Laboratory Methods}

Blood was collected for CA-125 analysis. Serum was separated within 4 hours using a refrigerated centrifuge. CA- 125 was estimated using CA-125 Elecsys kit based on ECLIA technology using Cobas e411 equipment (Roche, Germany).

\section{Evaluation of Ultrasound Data}

Size of large-sized unilocular ovarian cyst/s $(>5 \mathrm{~cm}$ ) without wall abnormalities were noticed from the ultrasound reports. Wherever more than one cyst was there, whether bilaterally or unilaterally, the size of all the cysts were added and the total volume was used for comparison with CA-125 levels.

\section{Statistical Analysis}

Data was analyzed using SPSS software, version 20.0 (Illinois, USA). The Kolmogorov-Smirnov test was done to assess the normal distribution of the variables. Correlation studies were performed to evaluate the relationship between different parameters. Spearman's Rho correlation was calculated for nonparametric data.

\section{Results}

Demographic, Clinical, CA-125 and Radiological Profile The recruited patients comprised 60 premenopausal subjects (mean age was $29.7 \pm 7.3$ years) ( - Table 1 ). The value of CA-125 in the study group was $118.0 \pm 147.1 \mathrm{IU} / \mathrm{mL}$, which was more than a three-fold increase than the baseline value of $\leq 35 \mathrm{IU} / \mathrm{mL}$. The ovarian cysts were present bilaterally in $51.6 \%$ subjects $(n=31)$ and unilaterally in $48.4 \%$ subjects $(n=29)$. All the patients had unilocular cysts and the mean size of cyst in all groups was $48.6 \pm 59.8 \mathrm{~mL}$, which was almost 10 -fold higher than the cutoff of $\leq 5 \mathrm{~cm}$ taken in this study. The profile of CA-125 and ovarian cyst size in different age groups is depicted in -Tables 2 and 3. 


\section{Analysis of Correlation between the CA -125 Levels and the Size of Ovarian Cysts}

Correlation analysis was performed by Spearman's Rho analysis using size of ovarian cyst in cubic centimeter (cc) and the CA 125 levels in IU/mL. No significant correlation was found between size of ovarian cysts and CA-125 levels $(R=0.005$, $p[2$-tailed $]=0.68)(-$ Table 4 and $\sim$ Fig. 1$)$.

Table 1 Demographic, tumor marker and radiological profile of the patients

\begin{tabular}{|l|l|l|}
\hline Mean age group* & Mean CA-125 levels* & $\begin{array}{l}\text { Mean ovarian } \\
\text { cyst volume* }\end{array}$ \\
\hline $29.7 \pm 7.3$ years & $118.0 \pm 147.1 \mathrm{IU} / \mathrm{mL}$ & $48.6 \pm 59.8 \mathrm{~mL}$ \\
\hline
\end{tabular}

*Expressed as mean and standard deviation.

Table 2 Profile of CA-125 levels in the different age groups

\begin{tabular}{|l|l|}
\hline Age group & CA-125 levels* \\
\hline $17-25$ years $(n=18)$ & $109.55 \pm 109.48$ \\
\hline $25-35$ years $(n=28)$ & $104.40 \pm 92.86$ \\
\hline $35-47$ years $(n=14)$ & $170.96 \pm 264.94$ \\
\hline All subjects $(n=60)$ & $119.61 \pm 147.95$ \\
\hline
\end{tabular}

*Expressed as mean and standard deviation.

Table 3 Profile of ovarian cyst volume in different age groups

\begin{tabular}{|l|l|}
\hline Age group & Ovarian cyst volume* \\
\hline $17-25$ years $(n=18)$ & $31.78 \pm 17.07$ \\
\hline $25-35$ years $(n=28)$ & $52.62 \pm 64.80$ \\
\hline $35-47$ years $(n=14)$ & $56.31 \pm 84.28$ \\
\hline All subjects $(n=60)$ & $48.67+59.81$ \\
\hline
\end{tabular}

*Expressed as mean and standard deviation.

\section{Discussion}

Ovarian carcinomas are the sixth most common cancers among women, and they are the seventh leading cause of death. In the Indian scenario, ovary is the third leading site for cancer after cervix and breast. ${ }^{8}$ Ovarian cancers have worst prognosis among all gynecological cancers and this is mostly due to their late diagnosis. Ovarian cancer remains asymptomatic in the early stages, which makes is difficult to diagnose them early. ${ }^{1}$ Noninvasive means for early diagnosis of ovarian cancer involves radiological imaging as well as biochemical markers. Imaging techniques used in diagnosis and monitoring of ovarian cancer have a wide spectrum, ranging from basic imaging modality like ultrasound to high-end techniques such as positron emission tomography (PET)-CT scan. Ultrasound is the most easily available noninvasive investigation, which is largely based on the operator skill. Higher imaging modality like multidetector CT scan provides speedy scan of entire body and is a good preoperative evaluation technique. MRI overcomes the limitations of CT scan like lack of lymph node visualization, with accuracy and spread of ovarian tumor in pelvis. PET CT scan plays an invaluable role in increasing staging accuracy by visualizing infra and supra diaphragmatic lymph nodes, and it is by far the best means available to identify residual or recurrent disease. ${ }^{8}$

Table 4 Correlation between analysis ovarian cyst volume and CA-125 levels

\begin{tabular}{|l|l|l|}
\hline Age group & Rvalue & $p$ value (2-tailed) \\
\hline $17-25$ years $(n=18)$ & 0.373 & 0.126 \\
\hline $25-35$ years $(n=28)$ & -0.032 & 0.870 \\
\hline $35-47$ years $(n=14)$ & -0.097 & 0.762 \\
\hline All subjects $(n=60)$ & 0.005 & 0.680 \\
\hline
\end{tabular}

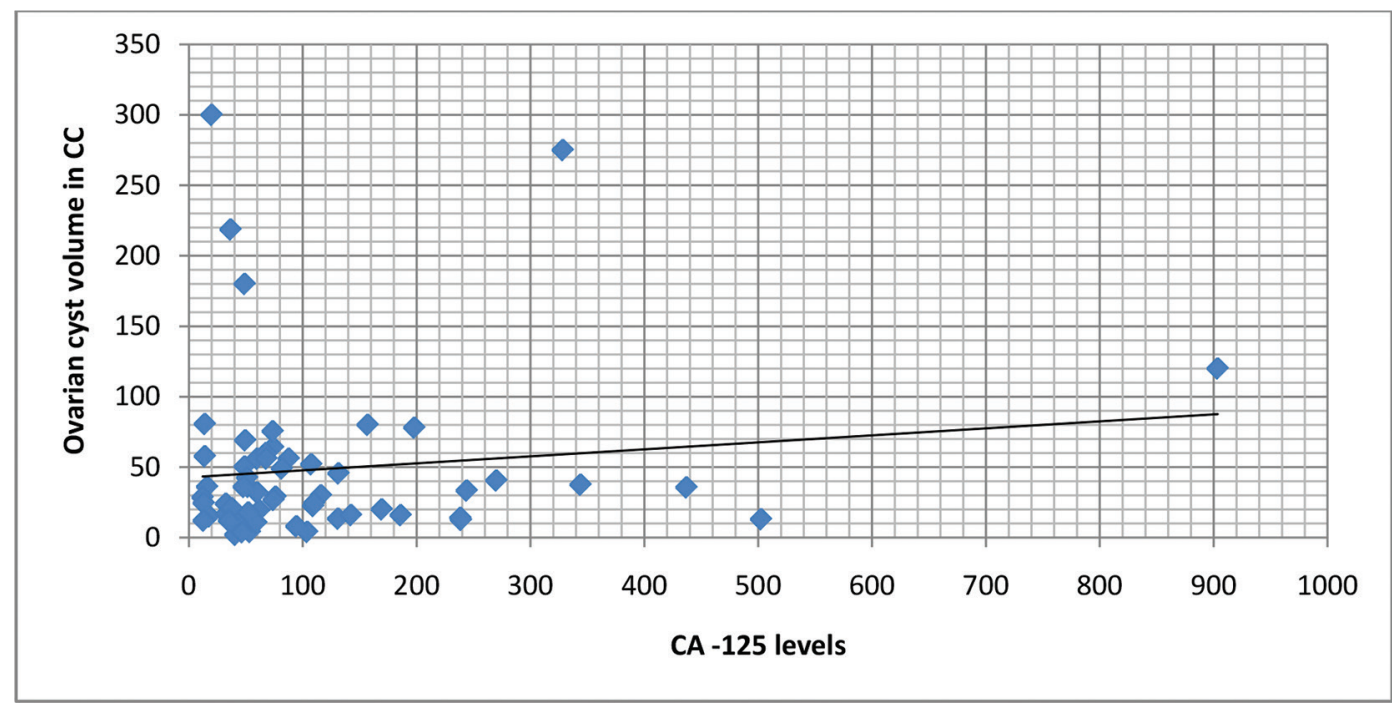

Fig. 1 Correlation analysis between ovarian cyst volume and CA-125 levels. 
Biomarkers used in ovarian cancer diagnosis include CA125, which is the best-known serum ovarian cancer biomarker; however, it has the drawback of less sensitivity and specificity. Other biochemical biomarkers are found to be more specific for certain ovarian tumors such as CEA (mucinous tumor) LDH (dysgerminoma, mixed germ cell tumors), $\alpha$-fetoprotein (embryonal cell tumors, yolk sac tumor), $\beta$-hCG (choriocarcinoma), inhibin B (granulosa cell tumors), and HE4 (Human epididymis protein 4). ${ }^{9}$ In this study, we evaluated CA-125 as the marker, since it is the most commonly done first-line tumor marker for screening ovarian malignancies.

Since neither biomarkers nor imaging alone is sufficient to diagnose ovarian cancer early, many studies have been done to evolve algorithms, in order to diagnose ovarian cancer based on serial radiological assessment or measurement of biomarkers or combination of both. ${ }^{10,11,12}$

In this context, Olivier et al had found that CA-125 combined with transvaginal ultrasound (TVUS) had positive predictive value of $40 \%$ for ovarian cancer; however, the diagnostic tool was found to be useful for diagnosing ovarian cancer in the advanced stage. ${ }^{12}$ Timmerman et al (2010) found that their logistic regression models based on information and gathered from ultrasound provided excellent discrimination between benign and malignant adnexal masses..$^{14} \mathrm{~A}$ follow-up study by Smith-Bindman et al, comprising 72093 women who had undergone pelvic ultrasound between 1997 and 2008, was conducted in the USA. They had concluded that those who had simple cysts on ultrasound did not develop ovarian cancer. ${ }^{13}$ However, most of the simple cysts they evaluated were of size less than $3 \mathrm{~cm}$. In this study, we tried to study, among Indian population, the malignant potential of largesized ( $>5 \mathrm{~cm}$ ) benign (simple) cysts. For this, we assessed the correlation of size of ovarian cysts with the CA-125 level in those patients who had raised level of CA-125 (> $35 \mathrm{IU} / \mathrm{mL}$ ). Our hypothesis was that even though CA-125 is not very specific for ovarian cancer, but the raised level of CA-125 in the presence of large-sized ovarian cyst may have the causeeffect relation between the two.

We found that there was no direct association found between CA-125 levels and size of ovarian cyst $(r=0.005, p=0.680)$.

In this regard, it is interesting to note that, as discussed above, CA-125 is a membrane glycoprotein which is found in the female genital tract of Mullerian duct origin (uterus, fallopian tube, upper part of vagina). Ovarian cystic epithelium is also of Mullerian origin; thus, expansion of cystic epithelium in larger cyst could have shown correlation with the CA-125 levels. ${ }^{5}$ But this lack of correlation may be due to what is now a well-accepted fact that high-grade sporadic epithelial ovarian tumors arise from serous tubal intraepithelial carcinoma (STIC) lesions of fallopian tube. Possibly, due to this, there was lack of direct correlation with ovarian cyst size. ${ }^{15}$

To diagnose the ovarian cancer early is key, as they are asymptomatic in the early stage. CA 125 is one such marker used for screening, but it is positive only in 20 percent cases. ${ }^{1}$ We need more evolved algorithms than RMI, as discussed above, to increase the early diagnostic efficacy of CA-125. In this study we tried to find correlation between ovarian cyst size and CA-125 level for this matter. Lack of correlation between size of ovarian cysts and CA-125 level, as found in this study, adds data to the ongoing quest in the scientific world to evolve mathematical models, in order to predict the possibility of neoplastic outcome in benign cysts with raised CA-125 levels.

We propose, therefore, in such cysts, which are benign in appearance but have a raised CA-125 levels, the fallopian tube epithelium should be evaluated radiologically or through PET-CT. Also, such cases should be evaluated further by other markers like CEA and CA 19.9 to rule out early malignancy.

\section{Strengths and Limitations of Our Study}

The strength of our study is that we included only premenopausal women with sonologically benign appearing ovarian cysts but having raised CA-125 levels and large size. This is a very less frequently studied group in the field of ovarian cancer research. Moreover, there is dearth of such data among Indian patients' group.

We chose CA-125 as a tumor marker for this study, as it is the most commonly ordered first-line tumor marker for screening ovarian cancer. But this was done at the cost of this marker being not highly specific for ovarian cancers only, as it is raised (up to $200 \mathrm{IU} / \mathrm{mL}$ ) in benign conditions like endometriosis, uterine fibroids, pancreatitis, pelvic inflammatory disease as well as cancers of colon, lung, breast and pancreas, although through clinical history and case record sheets, we tried our best to exclude these patients. Therefore, studying markers that are more specific for ovarian cancer like Human epididymis protein $4(\mathrm{He} 4)$ may have been better to assess the correlation of tumor marker with the size of benign ovarian cyst. Limitations of our study include a smaller number of patients and lack of follow-up to know the actual outcomes of these patients. Follow-up studies will better elucidate the progression of such cysts.

\section{Conclusion}

From our study, we conclude that benign ovarian cysts with raised CA-125 levels need evaluation of fallopian tubes to rule out ovarian cancer. Also, attention of the scientific community needs to be drawn toward exploring equations and algorithms for the early diagnosis of ovarian cancer, in order to assess correlation between ovarian cysts size and CA-125 levels, like this study.

\section{Ethical Clearance Statement}

The study protocol conformed to the ethical guidelines of the 1975 Declaration of Helsinki and was approved by the Institutional Ethical Committee for Human Research.

\section{Funding}

The project did not need any funding as it was carried out as part of patient care in the hospital.

\section{Conflicts of Interest}

The authors declares that there are no conflicts of interest. 


\section{Acknowledgments}

The author coveys his gratitude to the patients who participated in the study, the technical staff of the Department of Biochemistry, and hospital laboratory services at the Delhi State Cancer Institute, Delhi.

\section{References}

1 Farghaly SA. Current diagnosis and management of ovarian cysts. Clin Exp Obstet Gynecol 2014;41(6):609-612

2 Templeman C. Ovarian cysts. J Pediatr Adolesc Gynecol 2004; 17(4):297-298

3 Parazzini F, Frattaruolo MP, Chiaffarino F, Dridi D, Roncella E, Vercellini P. The limited oncogenic potential of unilocular adnexal cysts: a systematic review and meta-analysis. Eur J Obstet Gynecol Reprod Biol 2018;225:101-109

4 Dochez V, Caillon H, Vaucel E, Dimet J, Winer $\mathrm{N}$, Ducarme G. Biomarkers and algorithms for diagnosis of ovarian cancer: CA125, HE4, RMI and ROMA, a review. J Ovarian Res 2019;12(1):28

5 Nader R, Andrea H, Wittwer, CT, Tietz Fundamentals of Clinical Chemistry and Molecular Diagnostics. 1st south Asia ed. Philadelphia: Elsevier; 2018

6 Rockall A, Forstner R, Adnexal diseases. In: Hodler J, Kubik-Huch RA, von Schulthess GK, eds. Diseases of the Abdomen and Pelvis 2018-2021: Diagnostic Imaging - IDKD Book [Internet]. Cham (CH): Springer; 2018

7 Yang W-L, Lu Z, Bast RC Jr. The role of biomarkers in the management of epithelial ovarian cancer. Expert Rev Mol Diagn 2017;17(6):577-591
8 Fischerova D, Burgetova A. Imaging techniques for the evaluation of ovarian cancer. Best Pract Res Clin Obstet Gynaecol 2014;28(5):697-720

9 Ueland FR. A perspective on ovarian cancer biomarkers: past, present and yet-to-come. Available at: https://www.ncbi.nlm. nih.gov/pmc/articles/PMC5373023/. Accessed August 2, 2020

10 Ormsby EL, Pavlik EJ, van Nagell JR. Ultrasound follow up of an adnexal mass has the potential to save lives. Am J Obstet Gynecol 2015;213(5):657-661, 657.e1

11 Blyuss O, Burnell M, Ryan A, et al. Comparison of longitudinal CA125 algorithms as a first-line screen for ovarian cancer in the general population. Clin Cancer Res 2018;24(19):4726-4733

12 Olivier RI, Lubsen-Brandsma MA, Verhoef S. van Beurden M. CA125 and transvaginal ultrasound monitoring in high-risk women cannot prevent the diagnosis of advanced ovarian cancer. Gynecol Oncol 2006;100(1):20-26

13 Smith-Bindman R, Poder L, Johnson E, Miglioretti DL. Risk of malignant ovarian cancer based on ultrasonography findings in a large unselected population. JAMA Intern Med 2019; 179(1):71-77

14 Timmerman D, Van Calster B, Testa AC et al. Ovarian cancer prediction in adnexal masses using ultrasound-based logistic regression models: a temporal and external validation study by the 10TA group. Ultrasound obstet Gynecol 2010 36(2):226-234

15 Kumar V, Abbas AK, Aster J, Robbins \& Cotran Pathologic Basis of Disease. 10th ed. Philadelphia: Elsevier Saunders; 2017 